\title{
Is a timely assessment of the hematocrit necessary for cardiovascular magnetic resonance-derived extracellular volume measurements?
}

Mao-Yuan Su ${ }^{1,6+}{ }^{\mathbb{0}}$, Yu-Sen Huang ${ }^{1}$, Emi Niisato ${ }^{2 \dagger}$, Kelvin $\mathrm{Chow}^{3}$, Jyh-Ming Jimmy Juang ${ }^{4}$, Cho-Kai Wu ${ }^{4}$, Hsi-Yu Yu ${ }^{5}$, Lian-Yu Lin ${ }^{4}$, Shun-Chung Yang ${ }^{1}$ and Yeun-Chung Chang ${ }^{{ }^{*}}$

\begin{abstract}
Background: Cardiovascular magnetic resonance (CMR)-derived extracellular volume (ECV) requires a hematocrit (Hct) to correct contrast volume distributions in blood. However, the timely assessment of Hct can be challenging and has limited the routine clinical application of ECV. The goal of the present study was to evaluate whether ECV measurements lead to significant error if a venous Hct was unavailable on the day of CMR.

Methods: 109 patients with CMRT1 mapping and two venous Hcts (Hct 0 : a Hct from the day of CMR, and Hct, : a Hct from a different day) were retrospectively identified. A synthetic $\mathrm{Hct}\left(\mathrm{Hct}_{\text {syn }}\right)$ derived from native blood T1 was also assessed. The study used two different ECV methods, (1) a conventional method in which ECV was estimated from native and postcontrast T1 maps using a region-based method, and (2) an inline method in which ECV was directly measured from inline ECV mapping. ECVs measured with $\mathrm{Hct}_{0}, \mathrm{Hct}_{1}$, and $\mathrm{Hct}_{\text {syn }}$ were compared for each method, and the reference $E C V\left(E C V_{0}\right)$ was defined using the $\mathrm{Hct}_{0}$. The error between synthetic $\left(\mathrm{ECV}_{\text {syn }}\right)$ and $\mathrm{ECV}_{0}$ was analyzed for the two ECV methods.

Results: $\mathrm{ECV}$ measured using $\mathrm{Hct}_{1}$ and $\mathrm{Hct}_{\text {syn }}$ were significantly correlated with $\mathrm{ECV}_{0}$ for each method. No significant differences were observed between $\mathrm{ECV}_{0}$ and $\mathrm{ECV}$ measured with $\mathrm{HCt}_{1}\left(\mathrm{ECV}_{1} ; 28.4 \pm 6.6 \%\right.$ vs. $\left.28.3 \pm 6.1 \%, \mathrm{p}=0.789\right)$ and between $\mathrm{ECV}_{0}$ and $\mathrm{ECV}$ calculated with $\mathrm{Hct}_{\text {syn }}\left(\mathrm{ECV}_{\text {syn; }}\right.$ 28.4 $\pm 6.6 \%$ vs. 28.2 $\left.\pm 6.2 \%, \mathrm{p}=0.45\right)$ using the conventional method. Similarly, $E C V_{0}$ was not significantly different from $E C V_{1}(28.5 \pm 6.7 \%$ vs. $28.5 \pm 6.2, p=0.801)$ and $\mathrm{ECV}_{\text {syn }}(28.5 \pm 6.7 \%$ vs. $28.4 \pm 6.0, \mathrm{p}=0.974)$ using inline method. $\mathrm{ECV}_{\text {syn }}$ values revealed relatively large discrepancies in patients with lower Hcts compared with those with higher Hcts.

Conclusions: Venous Hcts measured on a different day from that of the CMR examination can still be used to measure ECV. ECV $_{\text {syn }}$ can provide an alternative method to quantify ECV without needing a blood sample, but significant ECV errors occur in patients with severe anemia.
\end{abstract}

Keywords: Cardiovascular magnetic resonance, Extracellular volume fraction, Hematocrit, T1 mapping

*Correspondence: ycc5566@ntu.edu.tw

${ }^{\dagger}$ Mao-Yuan Su and Emi Niisato have contributed equally to this work

${ }^{1}$ Department of Medical Imaging, National Taiwan University Hospital, No.7, Chung-Shan South Road, Taipei 100, Taiwan

Full list of author information is available at the end of the article

\section{Introduction}

The longitudinal relaxation time (T1) before and after the administration of contrast agents has been used to quantify the fraction of extracellular volume (ECV), which represents the extent of the extracellular space and can be used as a surrogate for quantifying diffuse myocardial original author(s) and the source, provide a link to the Creative Commons licence, and indicate if changes were made. The images or other third party material in this article are included in the article's Creative Commons licence, unless indicated otherwise in a credit line to the material. If material is not included in the article's Creative Commons licence and your intended use is not permitted by statutory regulation or exceeds the permitted use, you will need to obtain permission directly from the copyright holder. To view a copy of this licence, visit http://creativecommons.org/licenses/by/4.0/. The Creative Commons Public Domain Dedication waiver (http://creativeco mmons.org/publicdomain/zero/1.0/) applies to the data made available in this article, unless otherwise stated in a credit line to the data. 
fibrosis [1-4]. An ECV measurement requires a hematocrit (Hct) measurement to correct for contrast volume distributions in blood. The Society for Cardiovascular Magnetic Resonance (SCMR) Consensus Statement recommends that Hcts should be measured within $24 \mathrm{~h}$ of the CMR scan [5]. In many clinical practices, patients must have had a renal function evaluation within 3 months before cardiovascular magnetic resonance (CMR) with gadolinium contrast administration, from which a laboratory blood-derived (venous) Hct can be performed. In patients without laboratory bloodwork, an estimated glomerular filtration rate (eGFR) from blood drawn approximately 3 days before CMR can be alternatively used. To achieve accurate ECV measurements, a repeat venipuncture to acquire a Hct is performed on the day of CMR. Therefore, obtaining a venous Hct on the same day of the CMR is possible, but requires additional effort and increases the complexity of translating ECV quantification into routine clinical practice. As mentioned previously, Hct should be measured within $24 \mathrm{~h}$ of CMR as it may change over time. However, Hcts also vary with body posture [6] and diurnal fluctuation [7]. Studies have also demonstrated that Hct exhibits hour-to-hour, day-to-day, and even seasonal within-individual fluctuations $[8,9]$. It remains unknown whether these variations lead to significant errors in ECV measurements.

Blood consists of two water-containing compartments, erythrocytes and plasma, and the fraction of water in these two compartments is roughly proportional to their fractional volume and is referred to as the Hct. The longitudinal relaxation rate $(R 1=1 / T 1)$ in blood has been shown to be linearly correlated with Hct [10-13]. Previous studies have proposed that the "synthetic" Hct $\left(\mathrm{Hct}_{\text {syn }}\right)$ can be estimated from the native blood T1 values [14-16]. Treibel [17] and Fent [18] further demonstrated that synthetic ECVs $\left(\mathrm{ECV}_{\text {syn }}\right)$ derived from synthetically calculated Hcts $\left(\mathrm{Hct}_{\text {syn }}\right.$ ) demonstrated a strong correlation with ECVs derived from venous Hcts. However, several recent studies have suggested that using $\mathrm{ECV}_{\text {syn }}$ can result in the miscategorization of individual patients [19] and lead to clinical errors using 3T CMR [20]. Therefore, whether $\mathrm{ECV}_{\text {syn }}$ measurements using $\mathrm{Hct}_{\text {syn }}$ are feasible for clinical practice remains controversial and needs to be investigated further.

CMR-derived ECV can be evaluated using the region of interest (ROI)-based method either from native and postcontrast $\mathrm{T} 1$ maps or directly from ECV mapping. Whether the ECV value determined from the inline ECV mapping is comparable to the conventional $\mathrm{T} 1$ maps approach has yet to be established. Therefore, the goals of the present study were to evaluate: (1) whether significant ECV errors were found when the Hct was unavailable on the day of CMR, and (2) whether ECV values determined from the inline ECV method is comparable with that of the conventional ECV method.

\section{Methods \\ Patient populations}

This study was approved by the institutional review board. All the study participants provided written informed consent. Eligible patients underwent CMR and had two venous Hct performed: one measured on the same day of CMR $\left(\mathrm{Hct}_{0}\right)$ and the other measured on a different day $\left(\right.$ Hct $\left._{1}\right)$. Subjects with Hct $_{0}$ only were defined as the derivation group. The linear equation between blood $\mathrm{R} 1(1 / \mathrm{T} 1)$ and $\mathrm{Hct}_{0}$ was derived in the derivation group and used to estimate Hct $_{\text {syn }}$ and calculate a synthetic ECV. Exclusion criteria were uncontrolled arrhythmia, impaired renal function (eGFR $<30 \mathrm{ml} / \mathrm{min} / 1.73 \mathrm{~m}^{2}$ ), or contraindications to CMR (e.g., implanted devices). Clinical and demographic information, including underlying cardiac diagnoses, were collected.

\section{Imaging acquisition}

CMR was performed at 1.5T (Magnetom Aera, Siemens Healthineers, Erlangen, Germany) using a 30-channelbody coil and 32-channel spine coil. Myocardial T1 mapping was performed using an electrocardiogram (ECG)-triggered modified Look-Locker inversion recovery (MOLLI) pulse sequence, both before and 10-15 min after a $0.15 \mathrm{mmol} / \mathrm{kg}$ intravenous administration of a gadolinium-based contrast agent (Dotarem, Guerbet LLC, Princeton, New Jersey, USA). The MOLLI protocol used a 5(3)3 sampling scheme for native T1 mapping and a 4(1)3(1)2 sampling scheme for postcontrast T1 mapping. Scan parameters were as follows: TE/TR 1.14/2.7 ms; flip angle $35^{\circ}$; bandwidth $977 \mathrm{~Hz} / \mathrm{Px}$; minimum TI 125$150 \mathrm{~ms}$; TI increment $80 \mathrm{~ms}$; slice thickness $8 \mathrm{~mm}$; iPAT factor (GRAPPA) 2. Inline ECV mapping was automatically generated from the native and postcontrast T1 maps using an investigational prototype [22, 23]. Three inline ECV maps were reconstructed using venous Hcts $\left(\right.$ Hct $_{0}$, $\mathrm{Hct}_{1}$ ) and $\mathrm{Hct}_{\text {syn. }}$. Three evenly spaced short-axis slices were sequentially acquired from the left ventricular (LV) base to the apex.

\section{Imaging analysis}

Commercial post-processing software (cvi42, Circle Cardiovascular Imaging, Calgary, Canada) was used to analyze ECVs. A flowchart of ECV quantification is illustrated in Fig. 1. ECV, measured from native and postcontrast $\mathrm{T} 1$ maps using a region-based method, was defined as the conventional ECV method. The ROIs in the blood and myocardium of the LV were drawn in the central area of the LV cavity and the septal myocardium on the T1-mapping image at the middle slice. The average T1 


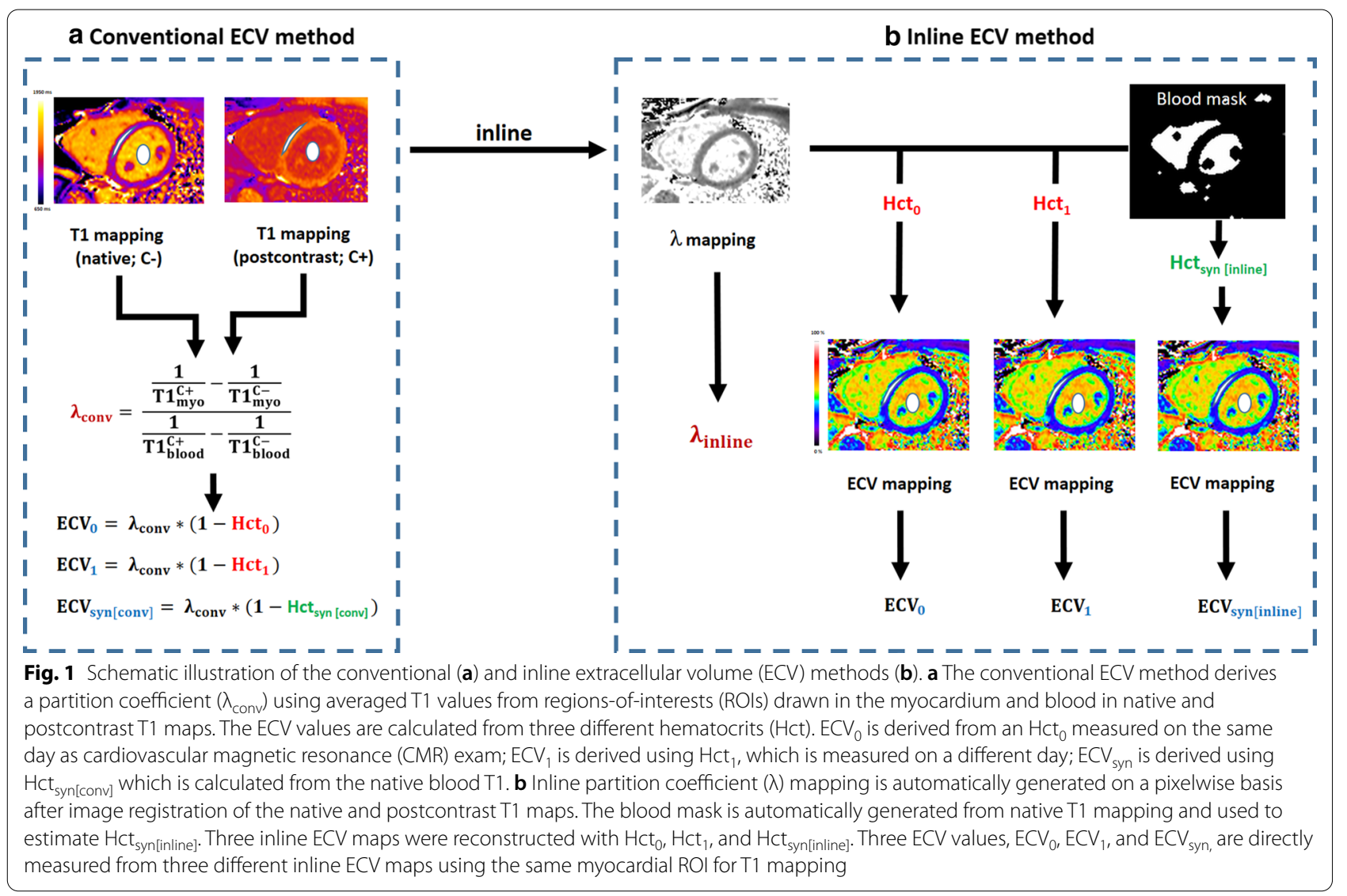

values of the segmented regions of interest were then computed. After obtaining the native and postcontrast T1 values, a partition coefficient $(\lambda)$ was calculated by using the following formula [24]:

$$
\lambda=\frac{\frac{1}{\mathrm{~T}_{\text {myocardium }}^{\text {postcontrast }}}-\frac{1}{\mathrm{~T} 1_{\text {myocardium }}^{\text {native }}}}{\frac{1}{\mathrm{~T}_{\text {blood }}^{\text {postcontrast }}}-\frac{1}{\mathrm{~T}_{\text {blood }}^{\text {native }}}}
$$

The ECV values were then obtained by multiplying $\lambda$ by 1 -Hct. For inline ECV mapping, the native and postcontrast T1 maps were aligned using non-rigid motion correction and the above formula was applied for each pixel, resulting in a $\lambda$ map. A $10 \%$ tolerance has been included for mismatching resolution/geometry, whereby the larger image was cropped to match the smaller image. The binary blood mask was automatically extracted using pre-defined thresholds and morphological imaging processing operations. The value for blood T1 was calculated as the median of all values of the T1 mapping identified by the blood mask. The native blood $\mathrm{T} 1$ was used to esti-

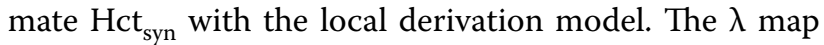
were scaled by $\mathrm{Hct}_{0}, \mathrm{Hct}_{1}$, and $\mathrm{Hct}_{\mathrm{syn}}$ to produce their respective ECV maps. Inline ECV values were measured directly from inline ECV maps using the same myocardial ROIs that were drawn on the T1 mapping in the conventional method (Fig. 1). The ECV measurement using $\mathrm{Hct}_{0}$ was defined as the reference ECV $\left(\mathrm{ECV}_{0}\right)$ for both the conventional and inline ECV methods. $\mathrm{ECV}_{0}$ was compared with ECV derived from $\mathrm{Hct}_{1}\left(\mathrm{ECV}_{1}\right)$ and the ECV derived from $\mathrm{Hct}_{\text {syn }}\left(\mathrm{ECV}_{\text {syn }}\right)$ for each method. $\mathrm{ECV}_{\text {syn }}$ error was defined by differences in ECV using $\mathrm{Hct}_{\text {syn }}$ and $\mathrm{Hct}_{0}$. The partition coefficient estimated from the conventional ECV method ( $\left.\lambda_{\text {conv }}\right)$ was compared with those estimated from the inline ECV method $\left(\lambda_{\text {inline }}\right)$. The relative changes in Hcts were assessed by comparing Hct $_{0}$ with $\mathrm{Hct}_{1}$ and $\mathrm{Hct}_{\text {syn }}$.

\section{Statistical analysis}

Continuous variables were expressed as the means and standard deviations (SDs) or as median (IQR; interquartile range) as appropriate, and categorical variables were expressed as percentages. Correlations between the continuous variables were assessed with the use of the Pearson correlation coefficient. Bias and precision were evaluated with Bland-Altman analyses. Agreements between the measurements were assessed via the 
intraclass correlation coefficient (ICC) with a two-way random-effects model. Comparisons between values were made using a paired $t$-test for continuous values with normal distributions and a Wilcoxon signed-rank test for continuous values with non-normal distributions. Statistical tests were two-tailed, and a statistical significance was defined as $p<0.05$. Type II error $(\beta)$ was calculated and statistical power was estimated by $1-\beta$ for each comparison. Equivalence analysis was performed to assess whether ECV estimated by $\mathrm{Hct}_{1}$ and $\mathrm{Hct}_{\text {syn }}$ were similar with $\mathrm{Hct}_{0}$ for each method. Sample size calculation for equivalence analysis was evaluated to achieve a $5 \%$ two sided type I error and $90 \%$ statistical power [25]. Two-sided 95\% confidential interval (CI) for the ECV difference between the two Hcts was used to compare with equivalence margin. Data were analyzed with SPSS (version 26, Statistical Package for the Social Sciences, International Business Machines, Inc., Armonk, New York, USA) and GraphPad Prism software (version 5.01, GraphPad Software, Inc., La Jolla, California, USA).

\section{Results}

\section{Patient characteristics}

812 consecutive patients were available for inclusion between March 2018 and May 2020. We excluded patients with suboptimal image quality $(n=12)$ and no contrast indication $(n=30)$ according to the exclusion criteria. A total of 770 subjects were included in this study. In this cohort, 37 patients without Hct data (5\% in total) were excluded. One hundred and ninetyfour patients had Hct data and CMR examinations performed on the same day (25.2\% in total). Among these patients, 85 patients without a second Hct were used to derive local derivation model for $\mathrm{Hct}_{\mathrm{syn}}$ and 109 patients who had a second Hct performed were included for further analysis (Fig. 2). The demographics of the study population are summarized in Table 1 . The time interval between the date of the second Hct and CMR was 117 days (IQR: 27-274 days). All patients were outpatient referrals. 24 patients (22\%) were hospitalized and 16 patients $(17 \%)$ underwent interventional procedures between the date of the second Hct and CMR. However, there was no significant difference in blood pressure and heart rate between these two time points. Clinical diagnoses consisted of amyloidosis $(\mathrm{n}=3,2.8 \%)$, Brugada syndrome $(n=6,5.5 \%)$, coronary artery disease $(n=12$, $11 \%)$, heart failure $(n=6,5.5 \%)$, dilated cardiomyopathy $(n=32.8 \%)$, Anderson-Fabry disease $(n=4,3.7 \%)$, hypertrophic cardiomyopathy $(\mathrm{n}=12,11 \%)$, myocarditis $(n=1,0.9 \%)$, hypertensive cardiomyopathy $(n=29,27 \%)$, tetralogy of Fallot $(n=6,5.5 \%)$, and suspected cardiovascular disease $(\mathrm{n}=18,17 \%)$.

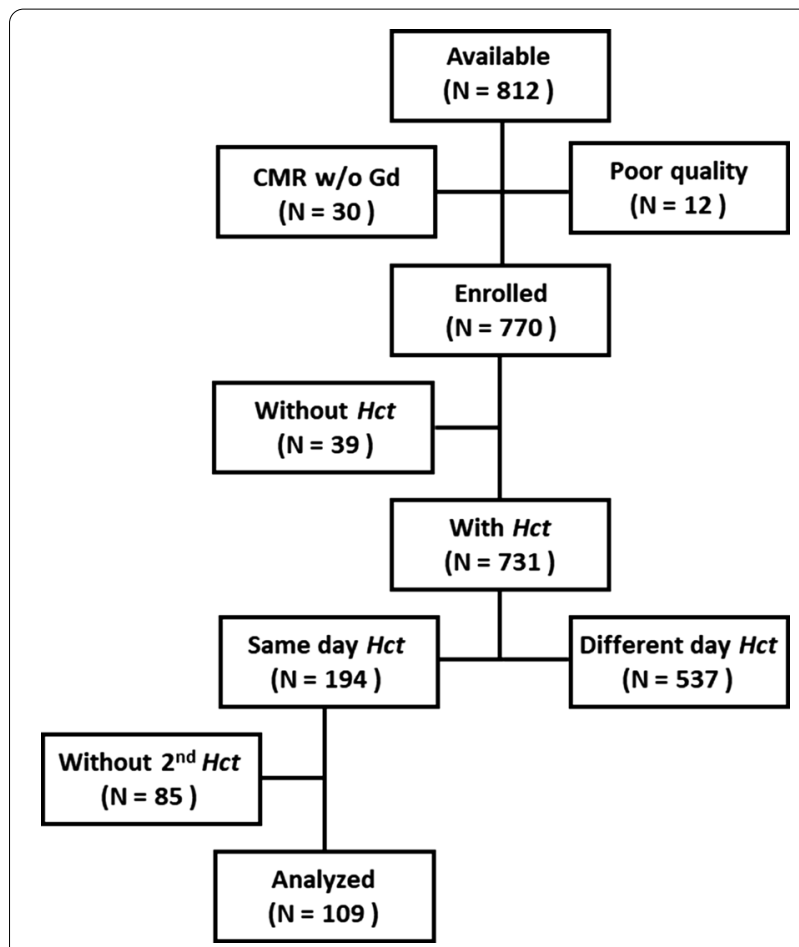

Fig. 2 A flow chart showing the selection process in this study. 812 consecutive patients were available for inclusion in this cohort. A total of 770 subjects were included in this study; 37 patients that did not have hematocrit (Hct) data were excluded. 149 patients had both Hct data and CMR performed on the same day. Among these patients, 109 patients had a second $\mathrm{Hct}\left(\mathrm{HCT}_{1}\right)$ measure and were included for further analysis

\section{Local derivation model for synthetic hematocrit}

The regression between $\mathrm{Hct}_{0}$ and native $\mathrm{T}_{\text {blood }}$ was linear $\left(R^{2}=0.51, p<0.001\right)$, and the regression equation was $\operatorname{Hct}_{\text {syn }}=\left[971.6^{*}\left(1 / \mathrm{T1}_{\text {blood }}\right)\right]+0.1818$ (Fig. 3a) in the derivation group.

\section{ECV comparisons with differently measured hematocrits and quantification methods}

Using the conventional method, ECVs measured with $\mathrm{Hct}_{1}$ and $\mathrm{Hct}_{\text {syn }}$ were significantly correlated with $\mathrm{ECV}_{0}$ (Fig. 4a). The coefficient of determination $\left(r^{2}\right)$ between $\mathrm{ECV}_{0}$ and $\mathrm{ECV}_{1}$ was $0.956(\mathrm{p}<0.001)$; the $r^{2}$ between $\mathrm{ECV}_{0}$ and $\mathrm{ECV}_{\text {syn }}$ was 0.935 ( $\left.\mathrm{p}<0.001\right)$. A Bland-Altman analysis indicated a $0.05 \%$ bias $(-2.8$ to $2.9 \%$, Fig. $4 \mathrm{c}$ ) between the $\mathrm{ECV}_{0}$ and $\mathrm{ECV}_{1}$ and a $0.2 \%$ bias (- 3.2 to $3.6 \%$, Fig. 4e) between the $\mathrm{ECV}_{0}$ and $\mathrm{ECV}_{\text {syn }}$. The ICC coefficient between $\mathrm{ECV}_{0}$ and $\mathrm{ECV}_{1}$ was 0.987 with a 95\% CI 0.991-0.982, and between $\mathrm{ECV}_{0}$ and $\mathrm{ECV}_{\text {syn }}$ was 0.981 with a $95 \%$ CI $0.987-0.973$. These results showed no significant differences between $\mathrm{ECV}_{0}$ and $\mathrm{ECV}_{1}$ $(28.4 \pm 6.6 \%$ vs. $28.3 \pm 6.1 \%, p=0.789, \beta=0.211)$ and 
Table 1 Patient characteristics

\begin{tabular}{|c|c|c|}
\hline & Nor mean $\pm S D$ & $\%$ or range \\
\hline Age (years) & $53 \pm 19$ & $17-89$ \\
\hline \multicolumn{3}{|l|}{ Gender } \\
\hline Male & 66 & 62 \\
\hline Female & 43 & 38 \\
\hline $\mathrm{BSA}\left(\mathrm{m}^{2}\right)$ & $1.73 \pm 0.25$ & $1.37-2.24$ \\
\hline Heart rate (bmp) & $74 \pm 15$ & $43-153$ \\
\hline \multicolumn{3}{|l|}{ LV function } \\
\hline EDVI $\left(\mathrm{ml} / \mathrm{m}^{2}\right)$ & $60.5 \pm 20.3$ & $32.0-156.9$ \\
\hline $\mathrm{ESVI}\left(\mathrm{ml} / \mathrm{m}^{2}\right)$ & $17.6 \pm 15.1$ & $3.3-101.9$ \\
\hline $\mathrm{EF}(\%)$ & $73 \pm 13$ & $33-93$ \\
\hline \multicolumn{3}{|l|}{ Diagnosis } \\
\hline Amyloidosis & 3 & 2.8 \\
\hline Brugada Syndrome & 6 & 5.5 \\
\hline Coronary artery disease & 12 & 11 \\
\hline Heart failure & 6 & 5.5 \\
\hline Dilated cardiomyopathy & 3 & 2.8 \\
\hline Anderson-Fabry disease & 4 & 3.7 \\
\hline Hypertrophic cardiomyopathy & 12 & 11 \\
\hline Hypertensive cardiomyopathy & 29 & 27 \\
\hline Myocarditis & 1 & 0.9 \\
\hline Tetrology of Fallot & 24 & 5.5 \\
\hline Others CVD & 18 & 17 \\
\hline
\end{tabular}

$B S A$ body surface area, EDVI end-diastolic volume index, ESVI end-systolic volume index, EF ejection fraction, $H c t$ hematocrit, $L V$ left ventricular, CVD cardiovascular disease

$\mathrm{ECV}_{0}$ and $\mathrm{ECV}_{\text {syn }}(28.4 \pm 6.6 \%$ vs. $28.2 \pm 6.2 \%, \mathrm{p}=0.450$, $\beta=0.536$; Fig. 4g).

Using the inline method, ECVs measured with Hct $_{1}$ and $\mathrm{Hct}_{\text {syn }}$ were also significantly correlated with $\mathrm{ECV}_{0}$ (Fig. 4b). The coefficient of determination $\left(r^{2}\right)$ between $\mathrm{ECV}_{0}$ and $\mathrm{ECV}_{1}$ measured with the inline ECV method was $0.957(\mathrm{p}<0.001)$; the $r^{2}$ between $\mathrm{ECV}_{0}$ and $\mathrm{ECV}_{\text {syn }}$ was $0.923(\mathrm{p}<0.001)$. Bland-Altman analysis indicated a $0.05 \%$ bias ( -2.73 to $2.82 \%$, Fig. $4 d$ ) between $E V_{0}$ and $\mathrm{ECV}_{1}$, and a $0.1 \%$ bias (- 3.58 to $3.85 \%$, Fig. $4 \mathrm{f}$ ) between $\mathrm{ECV}_{0}$ and $\mathrm{ECV}_{\text {syn }}$. The ICC coefficient between $\mathrm{ECV}_{0}$ and $\mathrm{ECV}_{1}$ was 0.988 with a 95\% CI 0.992-0.982, and between $\mathrm{ECV}_{0}$ and $\mathrm{ECV}_{\text {syn }}$ values was 0.977 with a 95\% CI 0.984-0.966. These results showed that in using the inline ECV method there was no significant difference between $\mathrm{ECV}_{0}$ and $\mathrm{ECV}_{1}(28.5 \pm 6.7 \%$ vs. $28.5 \pm 6.2$, $\mathrm{p}=0.801, \beta=0.199)$ and $\mathrm{ECV}_{0}$ and $\mathrm{ECV}_{\text {syn }}(28.5 \pm 6.7 \%$ vs. $28.4 \pm 6.0, p=0.974, \beta=0.026$ ) (Fig. 4 h).

In addition, the partition coefficient measured from the conventional ECV method was also not different with that measured from the inline ECV method ( $49.4 \pm 11.4 \%$ vs. $49.5 \pm 11.4 \%, p=0.620, \beta=0.378)$.

\section{Hematocrits compared on different sampling days and derived from native blood T1 mapping}

Bland-Altman analysis indicated a $0.03 \%$ bias (- 5.2 to $5.3 \%$ ) between $\mathrm{Hct}_{1}$ and $\mathrm{Hct}_{0}$ (Fig. $5 \mathrm{a}$ ), resulting in no significant difference between these two variables ( $42.3 \pm 4.7 \%$ vs. $42.4 \pm 4.7 \%, p=0.996, \beta=0.004$; Fig. $5 b)$. Regarding $\mathrm{Hct}_{\text {syn }}$ derived from the two different methods, Bland-Altman analysis indicated a $-0.4 \%$ bias ( -7.1 to $6.3 \%$ ) using the conventional ECV method (Fig. 5c) and a $-0.2 \%$ bias (- 7.2 to $6.9 \%$ ) using the inline ECV method (Fig. 5e). These results showed that Hct $_{\text {syn }}$ was not statistically different than Hct $_{0}$ using the conventional ECV method $(42.8 \pm 3.1 \%$ vs. $42.4 \pm 4.7 \%$, $\mathrm{p}=0.472, \beta=0.410$ ) (Fig. $5 \mathrm{~d}$ ) and using the inline ECV method $(42.5 \pm 2.8 \%$ vs. $42.4 \pm 4.7 \%, p=0.923, \beta=0.072$; Fig. 5f). Since the $\mathrm{Hct}_{\text {syn }}$ was determined from native blood T1 measurements, we further compared blood T1 measured using these two methods. From Bland-Altman
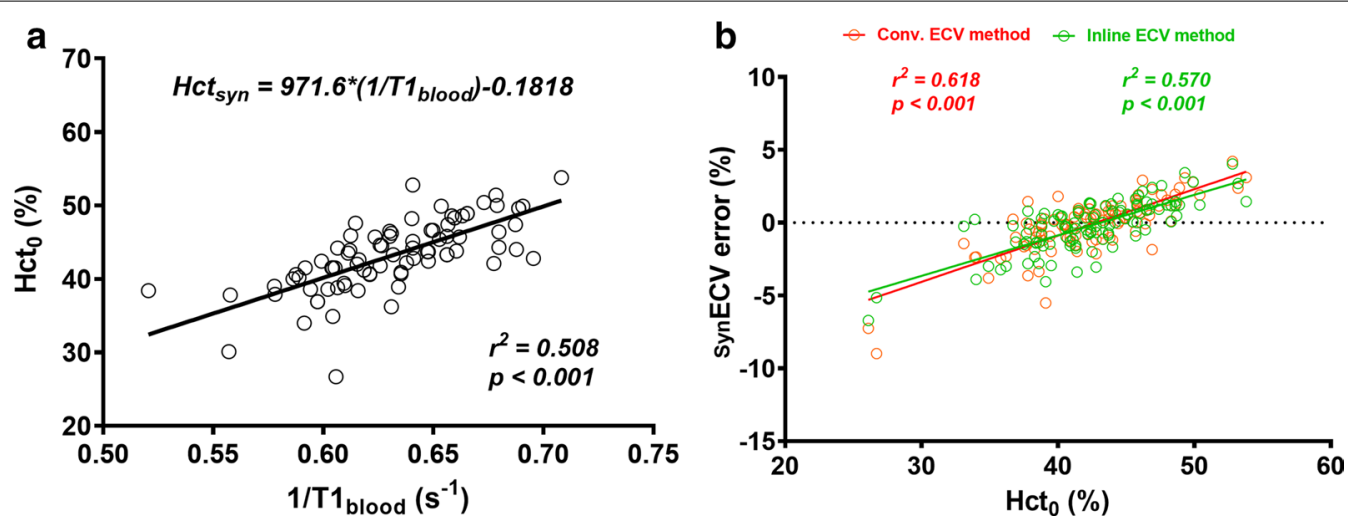

Fig. 3 a Linear regression between native blood 1/T1 (R1) and Hct measured on the same day as that of $\mathrm{CMR}(\mathrm{Hct})$ ). $\mathbf{b}$ Correlation graph comparing synthetic ECV error (synECV ${ }_{\text {error }}$ ) obtained from the conventional ECV method (red line) and the inline ECV method (black line). Both errors are positively associated with the Hct levels 
(See figure on next page.)

Fig. 4 Correlation and comparisons among three extracellular volumes (ECVs) derived from three different hematocrits (Hcts) using the conventional ECV and the inline ECV methods. $\mathrm{ECV}_{1}$ and $\mathrm{ECV}_{\text {syn }}$ are both strongly correlated with $\mathrm{ECV}_{0}$ in the conventional method (a) and the inline ECV method (b). In the conventional method, Bland-Altman plots indicate minimal bias between $\mathrm{ECV}_{1}$ and $\mathrm{ECV}_{0}(\mathbf{c})$ and between $\mathrm{ECV}_{\text {syn }}$ and $\mathrm{ECV}_{0}(\mathbf{e})$. Similarly, Bland-Altman plots also indicate minimal bias between $\mathrm{ECV}_{1}$ and $\mathrm{ECV}_{0}(\mathbf{d})$ and between $\mathrm{ECV}_{\text {syn }}$ and $\mathrm{ECV}_{0}(\mathbf{f})$ in inline $\mathrm{ECV}_{\text {method. }}$ No significant differences among $3 \mathrm{ECV}$ s in the conventional method $(\mathbf{g})$ and the inline $\mathrm{ECV}$ method (h) were observed. $\mathrm{ECV}_{0}$ is derived using an Hct obtained on the day of CMR (CMR; $\left.\mathrm{Hct}_{0}\right), \mathrm{ECV}_{1}$ is derived with $\mathrm{Hct}_{1}$ is measured from a different day than $\mathrm{CMR}_{\text {, and }} \mathrm{ECV}_{\text {syn }}$ is derived with $\mathrm{Hct}_{\text {syn' }}$ which is calculated from native blood T1 mapping

analysis, there was a $1.5 \mathrm{~ms}$ bias and confidence limit (7.2 to $6.9 \mathrm{~ms}$ ) using the inline method compared with using the conventional method (Fig. 5g), which resulted in no statistical difference between the conventional and inline ECV methods for native blood T1 measurements $(1599 \pm 84 \mathrm{~ms}$ vs. $1598 \pm 75 \mathrm{~ms}, \mathrm{p}=0.648, \beta=0.351$; Fig. 5h). Hct comparsions were listed in Table 2.

\section{Equivalence analysis for ECV differences}

Normal myocardial ECV in normal subjects has been reported at $1.5 \mathrm{~T}$ as $25.3 \pm 3.5 \% \quad(\mathrm{n}=81) \quad$ [26] and $25.4 \pm 2.5 \%(n=62)$ [23], respectively. Therefore, $a \pm 2 \%$ defined equivalence margin was considered acceptable in this study. Assuming SD of the ECV difference between the two Hcts was 4\%, 207 total samples were needed to achieve a 0.90 power with $\alpha=0.05$ for the equivalence study. The two-sided $95 \% \mathrm{CI}$ was calculated as followed:

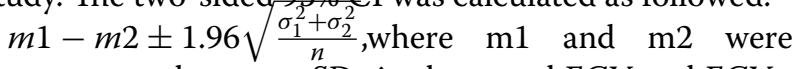
means; $\sigma_{1}$ and $\sigma_{2}$ were SDs in the tested ECV and $\mathrm{ECV}_{0}$, respectively, and the $\mathrm{n}$ was the sample size $(\mathrm{n}=109)$. Figure 6 showed the entire $\mathrm{CI}$ of ECV differences were within the equivalent margin in both Hct $_{1}$ and Hct $_{\text {syn }}$ for each method.

\section{Synthetic extracellular volume error is associated with hematocrit levels}

The error associated with $\mathrm{ECV}_{\text {syn }}$ measurements obtained from the two methods is shown in Fig. 6. Both errors were positively associated with $\mathrm{Hct}_{0}$ levels, which suggest that $\mathrm{ECV}_{\text {syn }}$ could be underestimated at lower Hcts and overestimated at higher Hcts. Based on a subanalysis of current data, the cut-off value for $\mathrm{Hct}_{\text {syn }}$ was determined on the error of $\mathrm{ECV}_{\text {syn }}$ was less than $2 \%$. The cut-off range for $\mathrm{Hct}_{\text {syn }}$ was obtained from 36.5 to $49.0 \%$ using the conventional method, and from 36.0 to $50.4 \%$ using the inline method, respectively.

\section{Discussion}

In this study, we compared ECV measurements with three different Hcts and two ECV methods. ECV measured with venous Hct drawn on a different day from that of CMR $\left(E C V_{1}\right)$ was significantly correlated with reference $\mathrm{ECV}\left(\mathrm{ECV}_{0}\right)$ using both conventional and inline
ECV methods. Minimal biases and limited discrepancies were also found. These findings demonstrate that for both methods, venous Hcts could be used to estimate $\mathrm{ECV}$, and that it did not matter if the Hct was from the day of CMR or from a different day. In addition, a significant correlation and good agreement between $\mathrm{ECV}_{\text {syn }}$ and $\mathrm{ECV}_{0}$ were noted in both methods. These results suggest that ECVs estimated with native blood T1 could be used for an ECV measurement if venous Hct was unavailable. Moreover, the equivalence analysis indicated that $95 \%$ CI of ECV differences between the two Hcts lay entirely within the equivalent margin for both methods. These results further strengthen our findings. The partition coefficient determined from the inline ECV method showed no significant difference with that of the conventional ECV method, suggesting that ECV measured using the inline method is reliably compared with ECV measured using the conventional method. Hct are necessary for ECV calculations and have been reportedly shown to be influenced by body posture [6] and diurnal fluctuation [7]. Some studies have also demonstrated that hemoglobin exhibits hour-to-hour, day-to-day, or even seasonal within-individual fluctuations $[8,9]$. Thirup et al. [27] performed a meta-analysis to explore the substantial variation of Hcts looking at 12 studies representing 638 healthy adults that had sampling intervals of 1 day to 1-2 months. They reported that both normal within-subject variation and analytical variation were $3 \%$, resulting in an approximately $12 \%$ relative change between the two successive Hct measurements. Theoretically, a $1 \%$ change in Hct would lead to a $0.67 \%$ change in the ECV. Based on this reference, a $12 \%$ change in Hct could cause an 8\% difference in the ECV. In this study, we demonstrated that there was no significant difference in the measured Hcts and ECVs using Hcts obtained on the same day vs. on a different day from the CMR, which could imply that the day on which an Hct is obtained does not have a significant effect on ECV measurements.

Hct $_{\text {syn }}$ can be derived from the native blood T1 values and used to calculate $\mathrm{ECV}_{\text {syn }}$ without needing blood sampling. Treibel et al. reported that $\mathrm{ECV}_{\text {syn }}$ measured from $\mathrm{Hct}_{\text {syn }}$ provide a validated, noninvasive quantification of the myocardial extracellular space without the need for a blood sample [17]. Fent et al. further demonstrated 

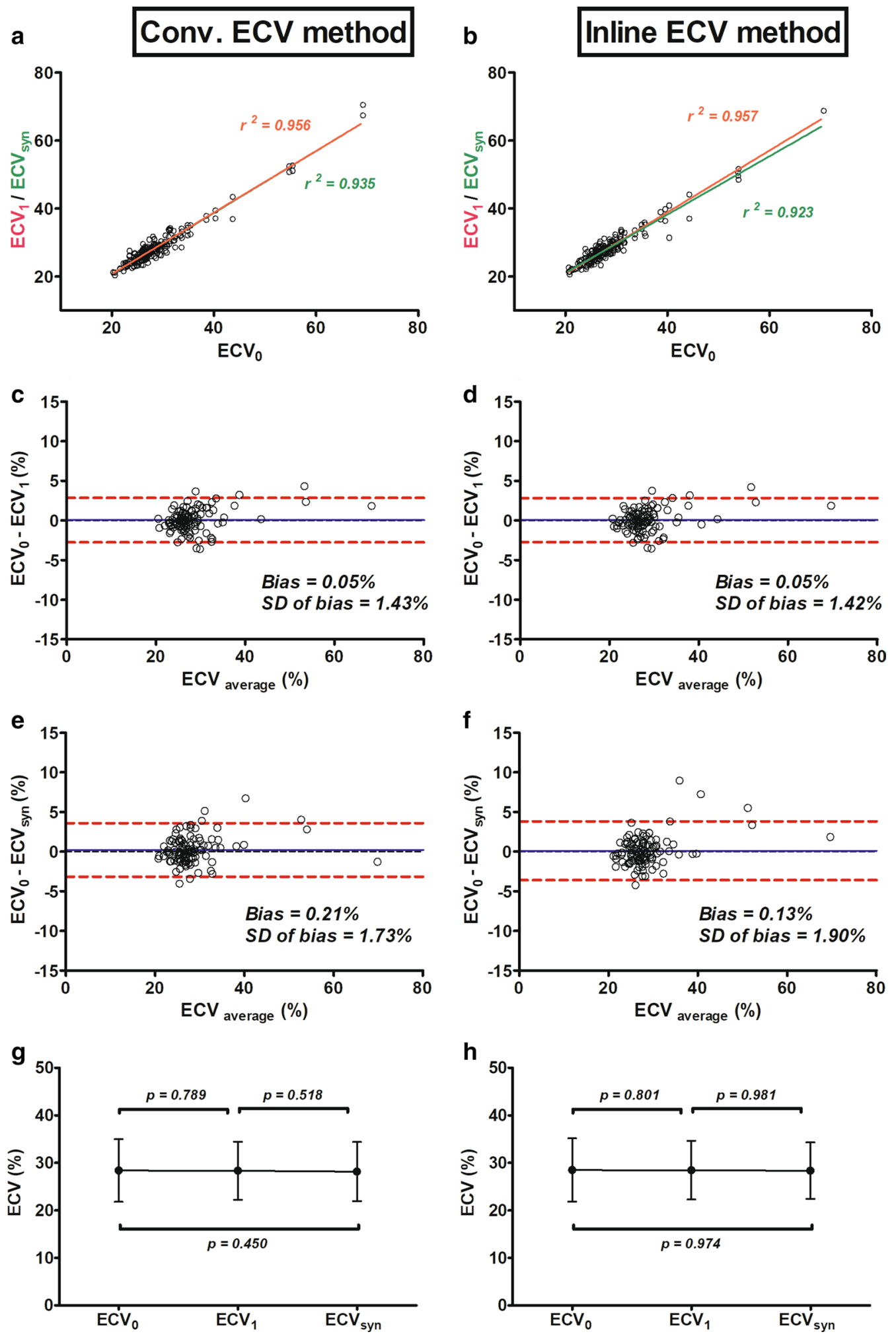


\section{(See figure on next page.)}

Fig. 5 Comparisons among three different hematocrits (Hcts) and between two native blood T1. Bland-Altman plots indicate the minimal bias between $\mathrm{Hct}_{1}$ and $\mathrm{Hct}_{0}(\mathbf{a})$, between $\mathrm{Hct}_{\text {syn [conv] }}$ and $\mathrm{Hct}_{0}(\mathbf{c})$, and between $\mathrm{Hct}_{\text {syn [inline] }}$ and $\mathrm{Hct}_{0}(\mathbf{e})$. There is no statistical difference between $\mathrm{Hct}_{1}$ and $\mathrm{Hct}_{0}(\mathbf{b})$, between $\mathrm{Hct}_{\text {syn [conv] }}$ and $\mathrm{Hct}_{0}(\mathbf{d})$, and between $\mathrm{Hct}_{\text {syn [inline] }}$ and $\mathrm{Hct}_{0}(\mathbf{f})$. Bland-Altman plot indicates a 1.5 ms bias and confidence limit (7.2-6.9 ms) using the inline method compared with using the conventional method ( $\mathbf{g}$ ), which resulted in no statistical difference between the conventional and inline ECV methods for native blood T1 measurements (h). Hct ${ }_{0}$ is Hct obtained on the same day as theCMR. Hct, is Hct obtained on a different day from the $\mathrm{CMR}_{\text {. }} \mathrm{Hct}_{\text {syn [conv] }}$ and $\mathrm{Hct}_{\text {syn [inline] }}$ are Hcts obtained synthetically from native blood T1mapping using the conventional and inline ECV methods, respectively

that $\mathrm{ECV}_{\text {syn }}$ values strongly correlated with conventionally measured ECV at both $1.5 \mathrm{~T}$ and $3 \mathrm{~T}$ [18]. In contrast, Raucci et al. proposed that using $\mathrm{ECV}_{\text {syn }}$ might result in a clinically significant miscategorization of pediatric and young adult patients [19]. In addition, Shang et al. further demonstrated that $\mathrm{ECV}_{\text {syn }}$ could lead to clinical errors using 3T CMR, suggesting that the use of $\mathrm{ECV}_{\text {syn }}$ could incorrectly categorize $6-25 \%$ of patients [20]. Consistent with some studies, our results showed that the $\mathrm{ECV}_{\text {syn }}$ was strongly correlated with the $\mathrm{ECV}_{0}$ and revealed no significant difference with $\mathrm{ECV}_{0}$ using both methods.

Our results showed that the native blood T1 values measured using the inline ECV method were not significantly different from those measured using the conventional ECV method. This finding suggests that automated blood region segmentation is reliable to estimate the blood T1 compared to conventional ROI method. Despite the blood T1 calculation, several factors that could influence native blood $\mathrm{T} 1$ are possible, such as physiologic variation (e.g., hemoglobin oxygenation [28], serum total proteins [29] and temperature), and technical issues (e.g., the efficiency of the inversion pulse, the pulse sequence parameters, the magnetization transfer effects, the magnetic field heterogeneity, and the fitting algorithms) [30-33]. In this study, we demonstrated that for the two ECV methods, $\mathrm{ECV}_{\text {syn }}$ error was significantly correlated with Hct, and the relatively large $\mathrm{ECV}_{\text {syn }}$ errors occurred when Hcts were lower compared with when they were higher. These findings suggest that the derived coefficients for Hct $_{\text {syn }}$ were only confidently applied to the range in which it was derived and extrapolations that occurred outside of the range resulted in less confident estimates. The patients with very low Hct values could have other blood composition abnormalities that also affect T1 measurements. For these patients, Hct $_{\text {syn }}$ and $\mathrm{ECV}_{\text {syn }}$ should be interpreted with caution, and a venous Hct should be used, if possible. For patients without a timely assessment of Hct, our results demonstrated that the last available Hct is feasible for use in ECV measurements. We defined the cut-off range (36-50\%) for $\mathrm{Hct}_{\text {syn }}$ based on the $\mathrm{ECV}_{\text {syn }}$ error of $<2 \%$. This range is similar with normal range of Hct: $41-50 \%$ for men and $36-48 \%$ for women. Although $\mathrm{ECV}_{\text {syn }}$ could lead to ECV error in patients with severe anemia, it also provides an alternative method to assess ECVs if venous Hcts are unavailable.

The purposes of CMR exams were diverse in this retrospective cohort. Patients with $\mathrm{Hct}_{0}$ were primarily intended for ECV evaluation. Therefore, only $25.2 \%$ of the enrolled CMR patients had Hct data from the same day as CMR, and $5 \%$ of patients had no Hct data before CMR. For our institution, two steps were required to obtain Hct $_{0}$ in our workflow. First, the laboratory bloodwork was requested with "fractional blood sampling", with one fraction for estimated eGFR and the other for Hct, so that two laboratory exams were tested simultaneously. Second, patients went to the laboratory department in advance to register for Hct evaluation and brought a blood collection tube to the CMR exam room. Then the blood sample was taken during the peripheral intravenous insertion by a CMR nurse before the exam. Due to number of additional steps required, this resulted in an $80 \%$ success rate for $\mathrm{Hct}_{0}$ collection in our experience. Common points of failure were the physician's assistant forgetting to remark "fractional blood sampling" on laboratory order sheet, the laboratory technologist omitting the request for two laboratory exams and blood sample not being taken during peripheral intravenous insertion. As Hct $_{0}$ collection is not part of our routine workflow, good communications between different departments and keeping patients well-informed were important for ensuring that Hct can be obtained on the same day as the CMR exam.

The conventional ECV method is most often used to quantify diffuse myocardial fibrosis. In these cases, the ROI can be drawn within the myocardium, avoiding the enhanced regions shown on late gadolinium enhancement (LGE) imaging. This method assumes that diffuse fibrosis is distributed homogeneously (uniform ECV) within the non-infarcted regions. However, the spatial variation of diffuse fibrosis is diverse and depends on various cardiomyopathies [34]. Therefore, ECV measurements could be affected by the position of an ROI if any significant regional variation exists in the fibrotic areas. Compared with conventional ECV method, inline ECV mapping not only allowed for ECV measurements at the time of CMR examination, but it also provided an ability to assess the heterogeneity of the myocardial tissue. This 

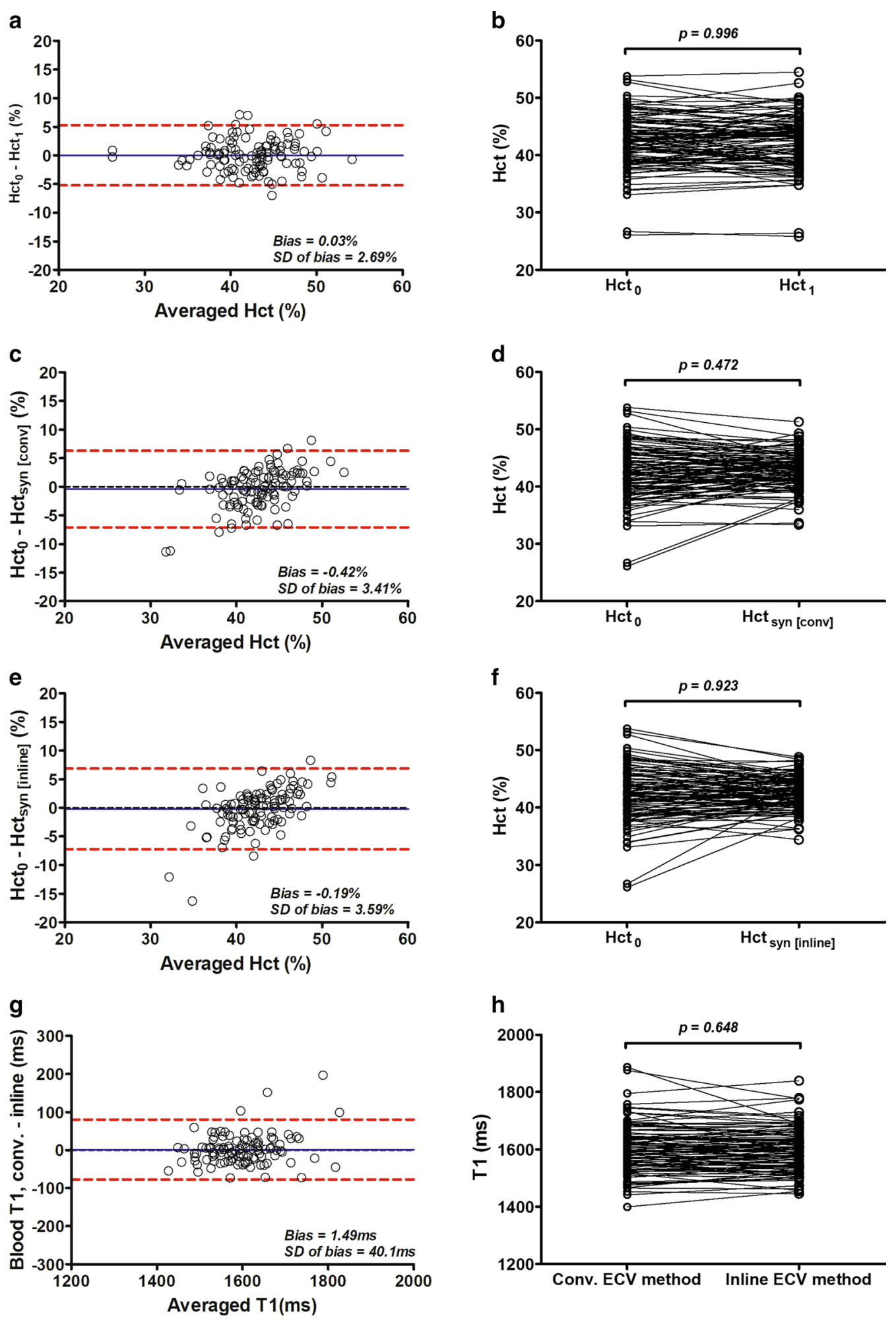
Table 2 Comparison of venous and synthetic hematocrits

\begin{tabular}{lll}
\hline & Mean \pm SD & Range \\
\hline $\mathrm{Hct}_{0}(\%)$ & $42.4 \pm 4.7$ & $(26.1,53.8)$ \\
$\mathrm{Hct}_{1}(\%)$ & $42.3 \pm 4.7$ & $(25.8,54.5)$ \\
$\mathrm{Hct}_{\text {syn [conv] }}(\%)$ & $42.8 \pm 3.1$ & $(33.4,51.3)$ \\
$\mathrm{Hct}_{\text {syn [inline] }}(\%)$ & $42.5 \pm 2.8$ & $(34.4,48.8)$ \\
\hline
\end{tabular}

$\mathrm{Hct}_{0}$ is hematocrit (Hct) obtained on the same day as that of $\mathrm{CMR}_{\text {. }} \mathrm{Hct}_{1}$ is $\mathrm{Hct}$ obtained on a different day from that of $\mathrm{CMR}$. $\mathrm{Hct}_{\text {syn [conv] }}$ and $\mathrm{Hct}_{\text {syn [inline] }}$ are Hcts obtained synthetically from native blood T1mapping using the conventional and inline ECV methods, respectively

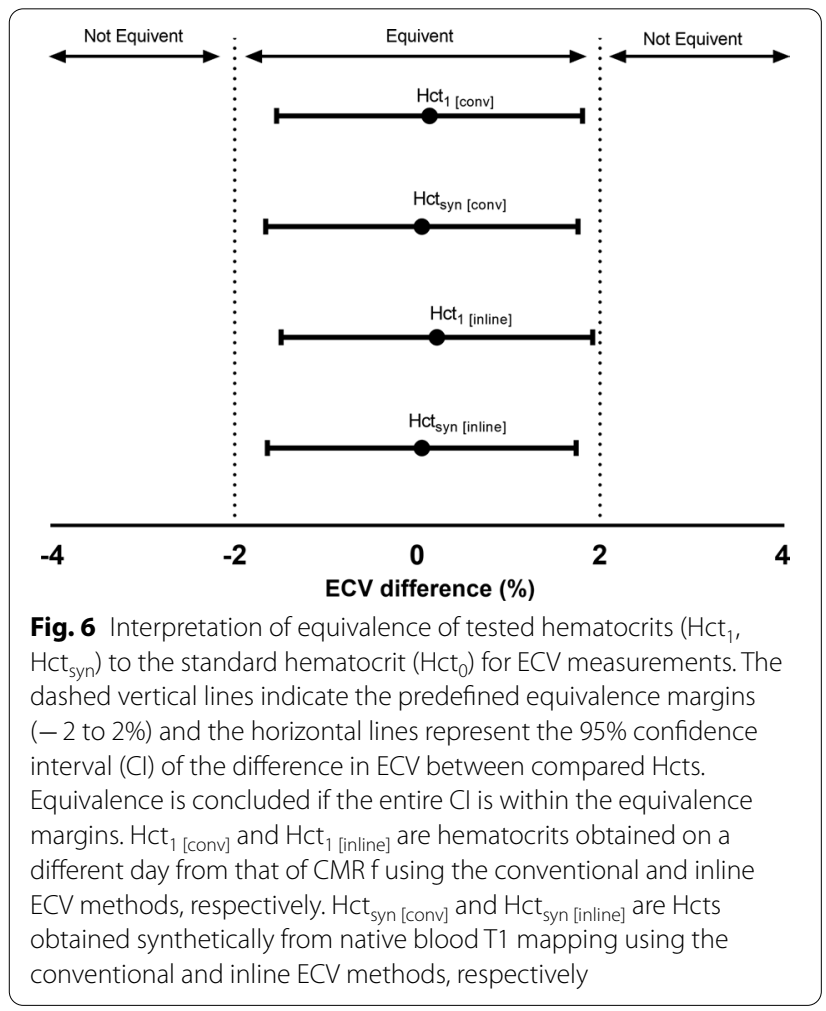

approach is clinically desirable and could potentially be used to identify subtle differences in myocardial ECVs earlier. In this study we demonstrated that the partition coefficient determined from the inline ECV method was not significantly different with that measured from the conventional ECV method. This result suggests that inline ECV method offers an identical ECV quantification compared to the conventional ECV method.

\section{Study limitations}

There are several limitations to our study. First, the ECVs measured using an Hct from a different day from that of CMR were not significantly different from those measured on the same day. This result could be associated with the lack of a statistical difference in the Hct depending on the time between when the measured Hct and CMR occurred. Although Hct has substantial variation, this variation does not appear to influence ECV measurements. Second, our study was performed with a single T1 pulse sequence (MOLLI), however different sequences of T1 mapping have been reported to yield different absolute ECV values [35]. Third, imaging quality for the ECV mapping was not evaluated in this study. Whether this comparison is identical with different pulse sequences or depends on the quality of ECV mapping is unknown and needs further investigation. Finally, all data were acquired at a single institution using a single CMR vendor. Multicenter studies, including larger numbers of patients, with different vendors should be further performed to validate these results.

\section{Conclusion}

Our study demonstrated that venous Hct measured on a different day than that of CMR is still useful for the calculation of ECVs regardless of the quantification method. $\mathrm{ECV}_{\text {syn }}$ values could provide an alternative method to quantify ECVs without requiring a blood sample but significant error may occur when patients have either extremely low or high Hcts. Inline ECV mapping could provide a method to quickly detect myocardial tissue heterogeneity and measure ECV abnormalities without the timely presence of a Hct.

\section{Abbreviations \\ CMR: Cardiovascular magnetic resonance; ECG: Electrocardiography; ECV: Extracellular volume fraction; ECV $V_{0}$ : Reference ECV; ECV ${ }_{\text {syn }}$ : Synthetic ECV; eGFR: Estimated glomerular filtration rate; $\mathrm{Hct}$ : Hematocrit; $\mathrm{Hct}_{0}$ : Reference hematocrit; $\mathrm{HCt}_{\text {syn: }}$ : Synthetic hematocrit; ICC: Intraclass correlation; LGE: Late gadolinium enhancement; LV: Left ventricle/left ventricular; MOLLI: Modified Look-Locker inversion recovery; ROI: Region of interest; SCMR: Society for Cardiovascular Magnetic Resonance; SD: Standard deviation; T1: Longitudinal relaxation time.}

\section{Acknowledgements}

NA

\section{Authors' contributions}

MYS and YCC are the guarantors of integrity of the entire study. MYS, EN, KC and YCC conceived the study, analyzed the CMR data and drafted the manuscript. LYL, CKW, HYY and JMJJ recruited the patients. MYS, EN and YSH acquired the CMR data and performed the statistical analyses. YSH, EN and YCC participated in the design and coordination of the study. EN and KC helped to edit the manuscript. All the authors read and approved the final manuscript.

\section{Funding}

This work was funded by the Ministry of Science and Technology, Taiwan (Grant Numbers MOST 106-2314-B-002-068).

\section{Availability of data and materials}

The datasets used and/or analyzed during the current study are available from the corresponding author upon reasonable request. 


\section{Ethics approval and consent to participate}

The study was approved by the IRB of the National Taiwan University Hospital, and all the participants provided written permission to participate in the study.

\section{Consent for publication}

Written informed consent was obtained from all the participants for publication of their individual details and images in this manuscript.

\section{Competing interests}

The authors declare that they have no competing interests.

\section{Author details}

${ }^{1}$ Department of Medical Imaging, National Taiwan University Hospital, No.7, Chung-Shan South Road, Taipei 100, Taiwan. ${ }^{2}$ Siemens Healthcare Limited, Taipei, Taiwan. ${ }^{3}$ Siemens Medical Solutions USA Inc., Chicago, IL, USA. ${ }^{4}$ Cardiovascular Center and Division of Cardiology, Department of Internal Medicine, National Taiwan University Hospital, Taipei, Taiwan. ${ }^{5}$ Department of Surgery, National Taiwan University Hospital, Taipei, Taiwan. ${ }^{6}$ Department of Medical Imaging and Radiological Technology, Yuanpei University of Medical Technology, Hsinchu, Taiwan.

Received: 5 June 2020 Accepted: 17 November 2020

Published online: 30 November 2020

\section{References}

1. Wong TC, Piehler K, Meier CG, et al. Association between extracellular matrix expansion quantified by cardiovascular magnetic resonance and short-term mortality. Circulation. 2012;126:1206-16.

2. Su MY, Lin LY, Tseng YH, et al. CMR-verified diffuse myocardial fibrosis is associated with diastolic dysfunction in HFpEF. JACC Cardiovasc Imaging. 2014;7:991-7.

3. Haaf P, Garg P, Messroghli DR, Broadbent DA, Greenwood JP, Plein S. Cardiac T1 Mapping and Extracellular Volume (ECV) in clinical practice: a comprehensive review. J Cardiovasc Magn Reson. 2016;18:89.

4. Rommel KP, von Roeder M, Latuscynski K, et al. Extracellular volume fraction for characterization of patients with heart failure and preserved ejection fraction. J Am Coll Cardiol. 2016;67:1815-25.

5. Messroghli DR, Moon JC, Ferreira VM, et al. Clinical recommendations for cardiovascular magnetic resonance mapping of T1, T2, T2* and extracellular volume: a consensus statement by the Society for Cardiovascular Magnetic Resonance (SCMR) endorsed by the European Association for Cardiovascular Imaging (EACVI). J Cardiovasc Magn Reson. 2017;19:75.

6. Jacob G, Raj SR, Ketch T, et al. Postural pseudoanemia: posture-dependent change in hematocrit. Mayo Clin Proc. 2005;80:611-4.

7. Sennels HP, Jorgensen HL, Hansen AL, Goetze JP, Fahrenkrug J. Diurnal variation of hematology parameters in healthy young males: the Bispebjerg study of diurnal variations. Scand J Clin Lab Invest. 2011;71:532-41.

8. Statland BE, Winkel P, Harris SC, Burdsall MJ, Saunders AM. Evaluation of biologic sources of variation of leukocyte counts and other hematologic quantities using very precise automated analyzers. Am J Clin Pathol. 1978;69:48-54.

9. Maes M, Scharpe S, Cooreman W, et al. Components of biological, including seasonal, variation in hematological measurements and plasma fibrinogen concentrations in normal humans. Experientia. 1995;51:141-9.

10. Silvennoinen MJ, Kettunen MI, Kauppinen RA. Effects of hematocrit and oxygen saturation level on blood spin-lattice relaxation. Magn Reson Med. 2003;49:568-71.

11. Lu H, Clingman C, Golay X, van Zijl PC. Determining the longitudinal relaxation time (T1) of blood at 3.0 Tesla. Magn Reson Med. 2004;52:679-82.

12. Shimada K, Nagasaka T, Shidahara M, Machida Y, Tamura H. In vivo measurement of longitudinal relaxation time of human blood by inversionrecovery fast gradient-echo MR imaging at 3T. Magn Reson Med Sci. 2012;11:265-71.

13. Grgac K, van Zijl PC, Qin Q. Hematocrit and oxygenation dependence of blood (1)H(2)O T(1) at 7 Tesla. Magn Reson Med. 2013;70:1153-9.

14. Braunschweiger PG, Schiffer L, Furmanski P. The measurement of extracellular water volumes in tissues by gadolinium modification of 1H-NMR spin lattice (T1) relaxation. Magn Reson Imaging. 1986;4:285-91.
15. Martin MA, Tatton WG, Lemaire C, Armstrong RL. Determination of extracellular/intracellular fluid ratios from magnetic resonance images: accuracy, feasibility, and implementation. Magn Reson Med. 1990;15:58-69.

16. Li W, Grgac K, Huang A, Yadav N, Qin Q, van Zijl PC. Quantitative theory for the longitudinal relaxation time of blood water. Magn Reson Med. 2016;76:270-81.

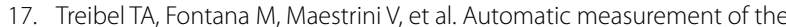
myocardial interstitium: synthetic extracellular volume quantification without hematocrit sampling. JACC Cardiovasc Imaging. 2016;9:54-63.

18. Fent GJ, Garg P, Foley JRJ, et al. Synthetic myocardial extracellular volume fraction. JACC Cardiovasc Imaging. 2017;10:1402-4

19. Raucci FJ Jr, Parra DA, Christensen JT, et al. Synthetic hematocrit derived from the longitudinal relaxation of blood can lead to clinically significant errors in measurement of extracellular volume fraction in pediatric and young adult patients. J Cardiovasc Magn Reson. 2017;19:58.

20. Shang $Y$, Zhang $X$, Zhou $X$, Wang J. Extracellular volume fraction measurements derived from the longitudinal relaxation of blood-based synthetic hematocrit may lead to clinical errors in $3 \mathrm{~T}$ cardiovascular magnetic resonance. J Cardiovasc Magn Reson. 2018;20:56.

21. Mewton N, Liu CY, Croisille P, Bluemke D, Lima JAC. Assessment of myocardial fibrosis with cardiovascular magnetic resonance. J Am Coll Cardiol [Internet]. 2011a;57:891-903.

22. Kellman P, Wilson JR, Xue H, Ugander M, Arai AE. Extracellular volume fraction mapping in the myocardium, part 1: evaluation of an automated method. J Cardiovasc Magn Reson. 2012;14:63.

23. Kellman P, Wilson JR, Xue $H$, et al. Extracellular volume fraction mapping in the myocardium, part 2: initial clinical experience. J Cardiovasc Magn Reson. 2012;14:64.

24. Jerosch-Herold M, Sheridan DC, Kushner JD, et al. Cardiac magnetic resonance imaging of myocardial contrast uptake and blood flow in patients affected with idiopathic or familial dilated cardiomyopathy. Am J Physiol Heart Circ Physiol. 2008;295:H1234-h1242.

25. Ahn S, Park SH, Lee KH. How to demonstrate similarity by using noninferiority and equivalence statistical testing in radiology research. Radiology. 2013;267:328-38

26. Sado DM, Flett AS, Banypersad SM, et al. Cardiovascular magnetic resonance measurement of myocardial extracellular volume in health and disease. Heart. 2012;98:1436-41.

27. Thirup P. Haematocrit: within-subject and seasonal variation. Sports Med (Auckland, NZ). 2003;33:231-43.

28. Atalay MK, Reeder SB, Zerhouni EA, Forder JR. Blood oxygenation dependence of T1 and T2 in the isolated, perfused rabbit heart at 4.7T. Magn Reson Med. 1995;34:623-7.

29. Jiao X, Bryant RG. Noninvasive measurement of protein concentration. Magn Reson Med. 1996;35:159-61.

30. Kawel N, Nacif M, Zavodni A, et al. T1 mapping of the myocardium: intraindividual assessment of the effect of field strength, cardiac cycle and variation by myocardial region. J Cardiovasc Magn Reson. 2012;14:27.

31. Kellman P, Arai AE, Xue H. T1 and extracellular volume mapping in the heart: estimation of error maps and the influence of noise on precision. J Cardiovasc Magn Reson. 2013;15:56.

32. Kellman P, Hansen MS. T1-mapping in the heart: accuracy and precision. J Cardiovasc Magn Reson. 2014;16:2.

33. Chow K, Flewitt JA, Green JD, Pagano JJ, Friedrich MG, Thompson RB. Saturation recovery single-shot acquisition (SASHA) for myocardial T(1) mapping. Magn Reson Med. 2014;71:2082-95.

34. Mewton N, Liu CY, Croisille P, Bluemke D, Lima JA. Assessment of myocardial fibrosis with cardiovascular magnetic resonance. J Am Coll Cardiol. 2011b;57:891-903.

35. Roujol S, Weingartner S, Foppa M, et al. Accuracy, precision, and reproducibility of four T1 mapping sequences: a head-to-head comparison of MOLLI, ShMOLLI, SASHA, and SAPPHIRE. Radiology. 2014;272:683-9.

\section{Publisher's Note}

Springer Nature remains neutral with regard to jurisdictional claims in published maps and institutional affiliations. 